\title{
Wie lange reicht die Energie?
}

\author{
Christine Kopp, Andreas M. Nieß
}

\begin{abstract}
In vielen Bereichen des Sports ist es vermeintlich von Nutzen, mit einem niedrigen Körpergewicht an den Start zu gehen. Doch eine solche Strategie birgt auch Risiken.
\end{abstract}

\section{Vorgeschichte}

In unserer sportmedizinischen Ambulanz stellte sich im September 2016 ein 14-jähriger Läufer zur Beratung bezüglich des weiteren Trainings vor. Üblicherweise erfolgt bei dieser Fragestellung eine sportmedizinische Grunduntersuchung, die eine allgemeine Anamnese, eine Trainingsanamnese, ein Ruhe-EKG sowie einen Laktatleistungstest beinhaltet. Fakultativ können eine Lungenfunktionstestung, eine Echokardiografie sowie eine Laboruntersuchung durchgeführt werden.

Im Anamnesegespräch erwähnte er im Beisein seiner Mutter, er habe keine Vorerkrankungen. Er befinde sich jedoch seit einigen Monaten in einer psychotherapeutischen Behandlung wegen seines geringen Gewichts. Zum Laufen kam er zufällig. Nach der erfolgreichen Teilnahme an einem Volkslauf wurde er im Ziel von einem Trainer als Talent gesichtet und angesprochen.

\section{Untersuchungsbefund}

14-jähriger Sportler, Körpergröße 162 cm, 42 kg, BMI $16 \mathrm{~kg} / \mathrm{m}^{2}$, Körperfettgehalt (Caliper-Methode) 4,4\%, kindliches Erscheinungsbild. Bei der körperlichen Untersuchung fanden sich bis auf das Untergewicht keine Auffälligkeiten. Das Ruhe-EKG und die Lungenfunktion waren ebenso wie die Echokardiografie regelrecht. In der Trainingsanamnese berichtete er über ein tägliches Lauftraining von bis zu 20 km/Einheit. Zusätzlich ging er schwimmen und nahm an einem Hockeytraining teil. Teilweise ging er in der großen Pause zum Laufen und absolvierte manchmal auch zwei Trainingseinheiten pro Tag. Infekte traten nicht gehäuft auf.

Bei den Laborwerten ergaben sich keine größeren Auffälligkeiten: Es zeigte sich eine diskrete Eisenmangelanämie; die Erhöhung von Kreatinkinase (CK) war auf das Training zurückzuführen.

In der Laufbandergometrie ( $\mathbf{A}$ Ab. 1) ergab sich eine für das Alter gute Leistungsfähigkeit. Was störte?!

\section{Procedere}

Der vorgestellte Sportler war untergewichtig, sein Wachstum war verzögert und es zeigte sich auch im Hinblick auf

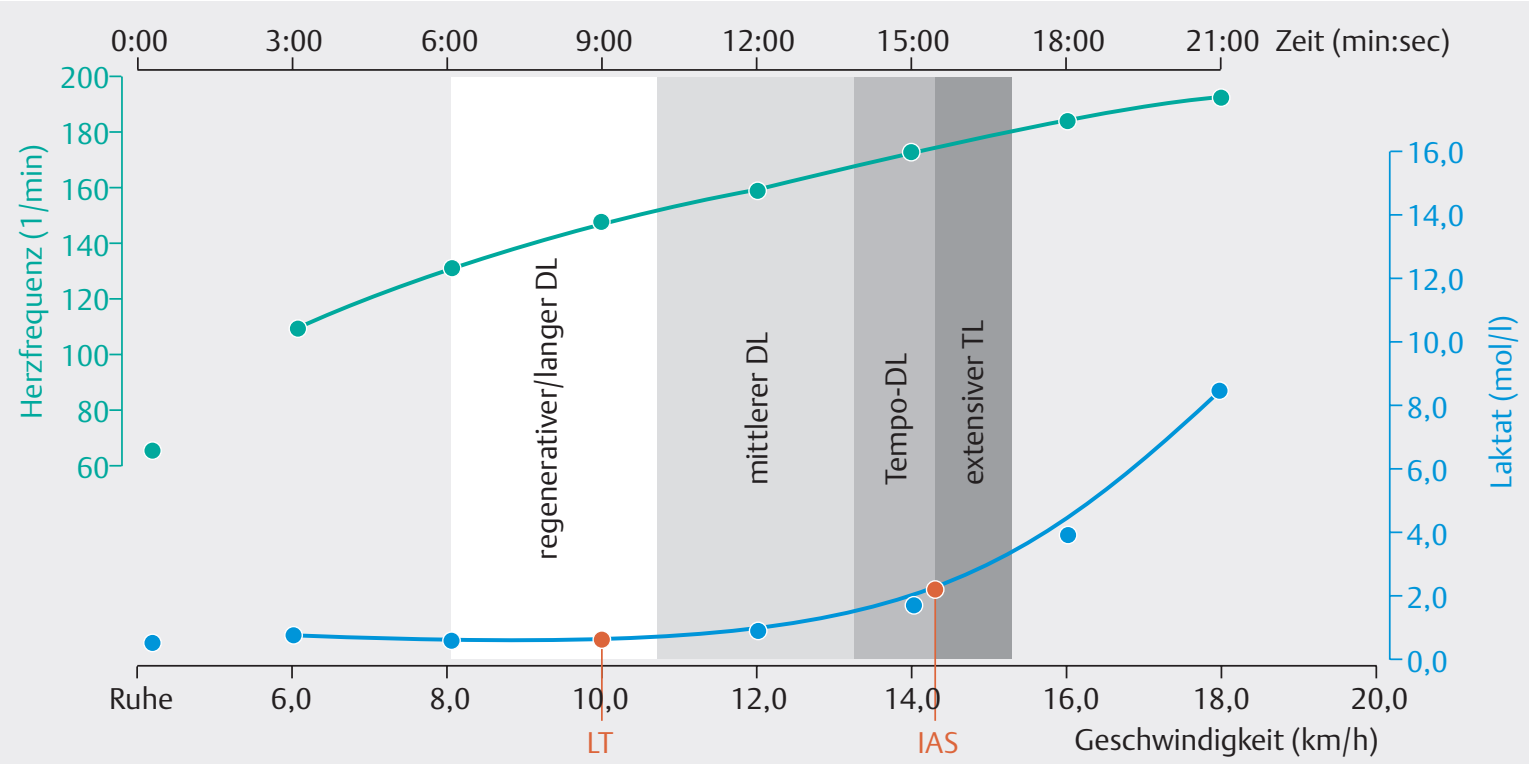

> Abb. 1 Laktatleistungskurve des jugendlichen Läufers zur Feststellung der körperlichen Leistungsfähigkeit (DL= Dauerlauf, TL = Tempolauf, LT = Laktatumschlagpunkt, IAS = individuelle anaerobe Schwelle) (Quelle: C. Kopp; grafische Umsetzung: Thieme Gruppe) 
sein Training ein auffälliges Verhalten. Daher wurde zunächst eine Trainingsreduktion empfohlen. Er sollte nicht mehr als dreimal wöchentlich trainieren. Zugleich wurde die Eisenmangelanämie mit einem Eisenpräparat behandelt. Bis auf Weiteres sollten monatliche Laborkontrollen beim Kinderarzt durchgeführt werden. Um das Gewicht zu steigern, erfolgte eine Ernährungsberatung, und der Jugendliche sollte sich einmal pro Woche in unserer Ambulanz zur Bestimmung der anthropometrischen Daten vorstellen. Unser Ziel war es, das bestehende Energiedefizit zeitnah auszugleichen. Zudem haben wir ein Wettkampfverbot ausgesprochen. Die bereits begonnene Psychotherapie sollte er wieder aufnehmen und Termine regelmäßiger wahrnehmen.

Bei der Therapieplanung haben wir uns an die Empfehlungen des IOC gehalten, die bei vorliegender Essstörung ein Wettkampfverbot und eine Trainingsreduktion sowie eine Wiederherstellung des Energiedefizits vorsehen [4].

\section{LINK ZU NÜTZLICHEN INFORMATIONEN ZU RED-S}

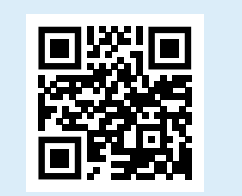

Das IOS Consensus Statement zu RED-S von 2014 findet man unter http://bit.ly/BTS-RED-S.

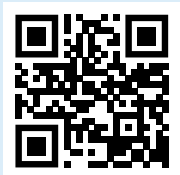

Zum Clinic Assessment Tool bei RED-S führt folgender Link: http://bit.ly/RED-S-CAT.
Die regelmäßigen Vorstellungen wurden gut eingehalten; der Sportler versuchte immer wieder, durch verschiedene Tricks seinen Trainingsumfang zu erhöhen. Das regelmäßige Wiegen und die Entwicklung des Körperfettes entwickelten sich zunächst erfreulich ( $\triangleright$ Tab. 1).

Ab April veränderte sich die Situation. Der Sportler versuchte, alle beteiligten Personen gegeneinander auszuspielen. Er wollte in ein Trainingslager gehen, die Teilnahme war jedoch an bestimmte Vorgaben geknüpft: stabiles Gewicht, regelmäßiges Essen und Trinken, Fortsetzen der Psychotherapie. Die wöchentlichen Vorstellungen waren von Streitigkeiten mit uns und seinen Eltern geprägt.

Um, auch im Sinne des Sportlers, eine Entscheidung bezüglich des Trainingslagers zu treffen, haben wir Mitte April bei uns die Laborwerte bestimmt. Dabei ergab sich ein verändertes Bild der Situation ( $\vee$ Tab. 1).

Der Befund zeigte eine deutliche Hyponatriämie. Dies war eine Situation, die für uns die Entscheidung gegen die Teilnahme am Trainingslager leichter machte, denn

\section{HYPONATRIÄMIE}

Durch Verdünnung und/oder falsches Trinken kann es bei Sportlern zu einer Hyponatriämie kommen, insbesondere wenn hohe Außentemperaturen herrschen und der Sportler sehr schwitzt. 3\% der ungeklärten Todesfälle beim Marathon sind auf eine Erniedrigung des Natriums zurückzuführen ( $\bullet$ Abb.2a) [3]. Beim Trinken ist daher auf eine ausreichende Zufuhr von Natrium zu achten

( A Abb.2b). Dies kann auch durch leichtes Salzen der Getränke erreicht werden.

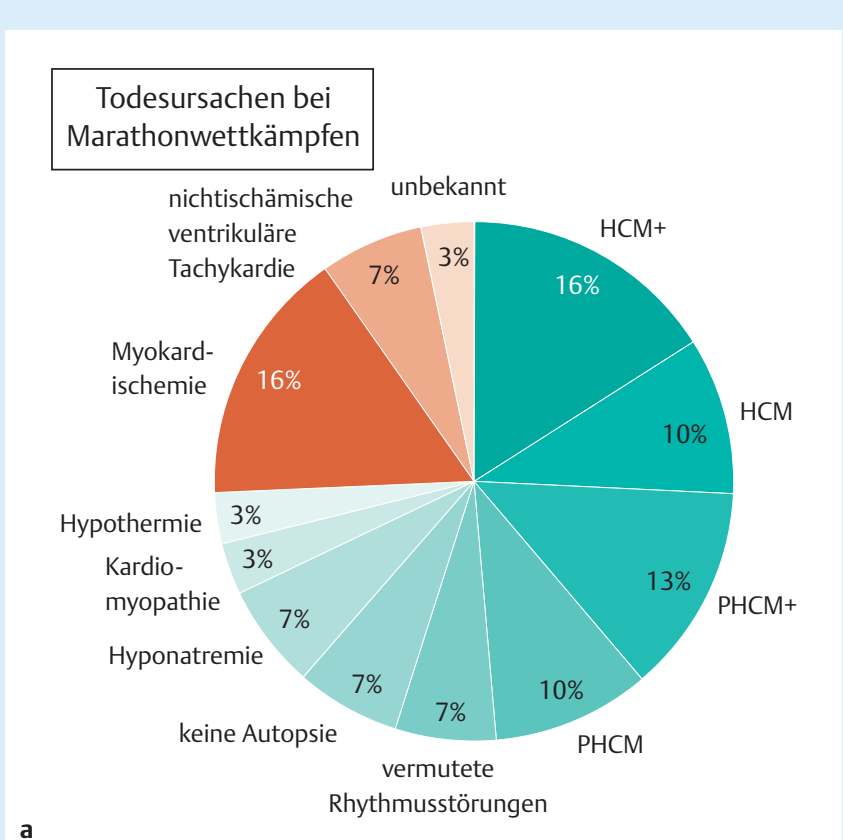

a

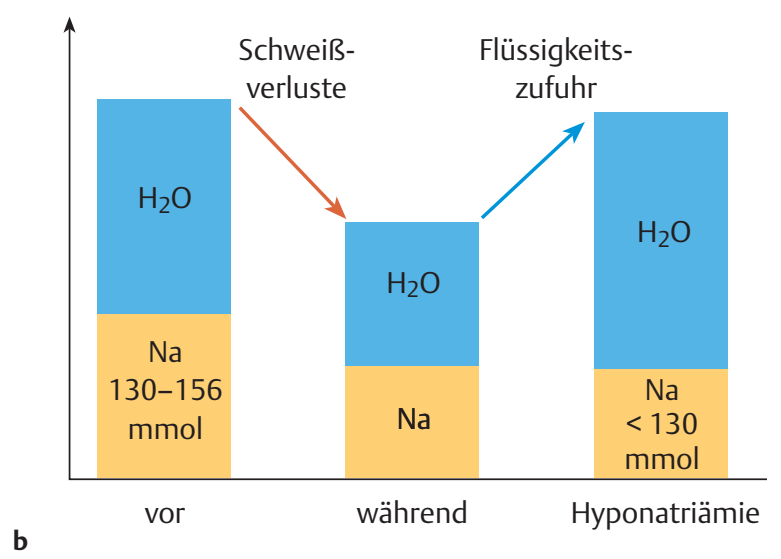

- Abb. 2 Hyponatriämie kann gefährliche Auswirkungen haben. a $3 \%$ der ungeklärten Todesfälle beim Marathon sind auf eine Erniedrigung des Natriums zurückzuführen (HCM = hypertrophe Kardiomyopathie, $\mathrm{PHCM}=$ mögliche hypertrophe Kardiomyopathie, + = zusätzliche Diagnosen wie z. B. Arteriosklerose der Herzkranzgefäße, Myokarditis etc.) (Quelle: Kim et al. 2012; grafische Umsetzung: Thieme Gruppe). b Wird der Flüssigkeitsverlust durch Schwitzen nicht mit natriumhaltigen Getränken ausgeglichen, kommt es u. U. zu eine Hyponatriämie (Quelle: C. Kopp; grafische Umsetzung: Thieme Gruppe). 
- Tab. 1 Veränderungen von Körpergewicht, Körperfett und Natriumkonzentration des jugendlichen Läufers im Verlauf der Therapiesitzungen

\begin{tabular}{|l|l|l|l|}
\hline Datum & Gewicht in $\mathbf{k g}$ & Körperfett in \% & $\begin{array}{l}\text { Elektrolyte } \\
\text { Natrium [mmol] }\end{array}$ \\
\hline $\mathbf{2 7 . 0 9 . 1 6}$ & $\mathbf{4 2 , 0}$ & $\mathbf{4 , 4}$ & $\mathbf{1 4 0}$ \\
\hline $\mathbf{1 8 . 1 0 . 1 6}$ & 43,7 & 6,2 & \\
\hline 22.11 .16 & 43,9 & 5,9 & \\
\hline 14.12 .16 & 44,8 & 5,3 & \\
\hline 30.01 .17 & 46,0 & 4,4 & 130 \\
\hline 20.02 .17 & 46,4 & 5,3 & 129 \\
\hline 23.03 .17 & 46,2 & 8,1 & 139 \\
\hline 06.04 .17 & 45,7 & 6,8 & \\
\hline 12.04 .17 & - & - & \\
\hline & & & \\
\hline
\end{tabular}

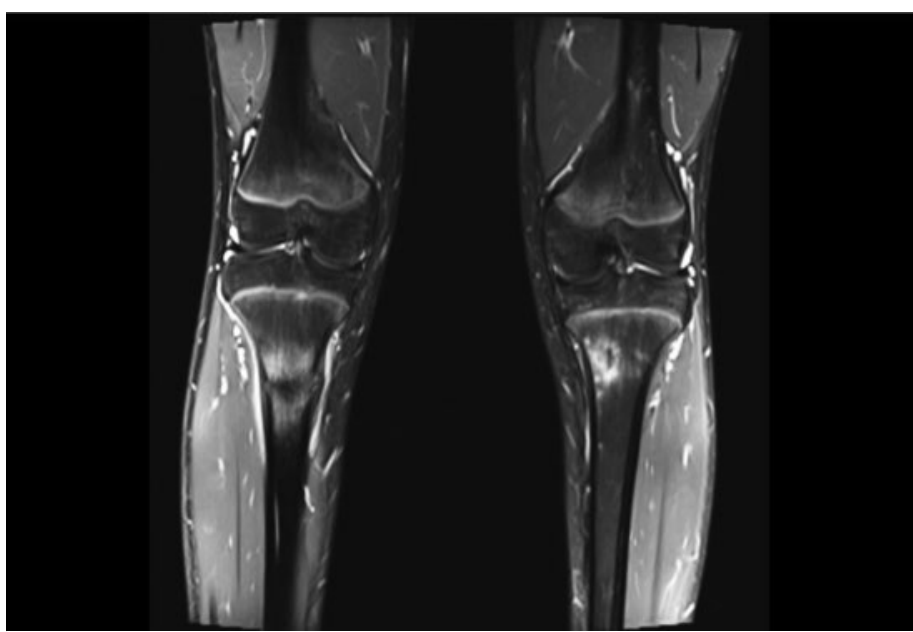

Abb. 3 Ermüdungsfraktur des linken proximalen Tibiaschaftes, beginnende Ermüdungsfraktur des rechten proximalen Tibiaschaftes (Quelle: C. Kopp) linken Tibia und eine beginnende Ermüdungsfraktur der rechten Tibia ( $\mathbf{A b b} \mathbf{3} \mathbf{3})$.

Somit war eine Trainingspause zwangsweise gegeben. Der Sportler hat in dieser Zeit seine Psychotherapie weitergeführt und im Verlauf auch an Gewicht zugelegt. Dabei ergab sich als erfreuliche Nebenerscheinung, dass er endlich auch etwas gewachsen ist.

Dieser Fall zeigt deutlich das Vorliegen eines RED-S-Syndroms (siehe hierzu > Abb. 1, S. 61 Einführungsartikel) mit allen potenziellen Begleiterkrankungen. Auch zeigt dieser Fall, dass nicht nur Frauen und Mädchen von diesem Symptomenkomplex betroffen sein können.

\section{Fazit}

In der Sportbetreuung sollte immer, speziell bei körperbetonten Sportarten, an das Vorliegen eines RED-S-Syndroms gedacht werden. Wichtig ist dabei, bereits in der Anamnese auf typische Hinweise zu achten. Hierzu gehören Trainingsumfänge, Infektanfälligkeiten, Angaben zur Regelblutung, Ermüdungsfrakturen sowie das Erheben von Ernährungsgewohnheiten[1]. Sollte sich der Verdacht bestätigen, ist es wichtig, im Team weiterzuarbeiten. In dieses Team gehören der Sportler, das Umfeld, der Trainer, der Manager, Ärzte wie Gynäkologe, Psychotherapeut/Psychiater und Ernährungsberater[4]. Man sollte sich nicht scheuen, auch mal gemeinsam ein Sportverbot auszusprechen. Das wichtigste Tool zur Gesundung ist das Abbauen des Energiedefizites. Das wird oft nur durch zeitweilige Sportreduktion erreicht [4]. In dieser Phase sollte der Sportler nicht allein gelassen werden, sondern er bedarf einer guten und intensiven Betreuung. Sind die Symptome gravierend, muss auch eine stationäre Therapie erwogen werden.

Im Alltag ist es wichtig, dass der Sportler nicht durch unkluge Bemerkungen wie z. B. „Ein Kilo weniger macht dich eine Sekunde schneller “ in ein verändertes, gar pathologisches Essverhalten gedrängt wird. Sollte ein kritisches Verhältnis von Größe, Gewicht und Körperfett unterschritten werden, ist in der Regel auch mit einem Leistungseinbruch zu rechnen[5]. Das muss den Betroffenen auch ehrlich kommuniziert werden. Sich an die „Back to Sports “-Regeln des IOC zu halten, macht den Umgang mit betroffenen Athleten etwas einfacher, da viele ein Regelwerk von oberster Stelle eher anerkennen und man hiermit auch Perspektiven aufzeigen kann.

Nicht jeder Athlet mit einem Energiedefizit leidet zugleich an einer Essstörung; dies muss frühzeitig abgeklärt werden. 


\section{TAKE HOME MESSAGE}

- Untergewicht bei Sportlern ist nicht physiologisch.

- Eine primäre oder sekundäre Amenorrhoe muss gynäkologisch abgeklärt und ggf. therapiert werden; sie ist bei Leistungssportlerinnen nicht normal.

- Ermüdungsfrakturen müssen hellhörig machen, hier sollte eine Osteopenie ausgeschlossen werden[2].

- Häufige Infekte sind ein weiterer Hinweis und müssen ebenfalls abgeklärt werden.

- Bei Verdacht auf Vorliegen eines RED-S-Syndroms sollte multidisziplinär behandelt werden (Sportmedizin, Psychosomatik, betreuender Arzt, Gynäkologe, Endokrinologe).

- An erster Stelle der Behandlung steht der Ausgleich des Energiedefizites, um bleibende Schäden abzuwenden.

Autorinnen/Autoren

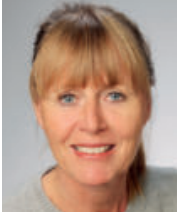

\section{Christine Kopp}

Dr. med. Christine Kopp ist Fachärztin für Allgemeinmedizin, Sportmedizin und Ernährungsmedizin. Sie ist Funktionsoberärztin an der Medizinischen Klinik am Universitätsklinikum Tübingen, Abteilung Sportmedizin und dort verantwortlich für Kadersportler. Als Verbandsärztin des Deutschen Leichtathletikverbands ist sie Mitglied in der medizinischen Kommission des DOSB.

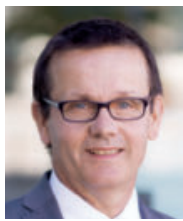

\section{Andreas M. Nieß}

Prof. Dr. med. Andreas Nieß, Facharzt für Innere Medizin und Sportmedizin, ist Ordinarius und ärztlicher Direktor der Abteilung Sportmedizin an der Medizinischen Klinik am Universitätsklinikum Tübingen. Seine Forschungsschwerpunkte sind zelluläre

Stressantwort und Anpassungen an akute und chronische sportliche Belastung, klinische (Trainings-)Interventionsstudien sowie die Gesundheitsförderung im Leistungssport. Klinische Schwerpunkte sind sportmedizinische Untersuchungen und Trainingstherapie bei internistischen Erkrankungen. Er ist Mitglied verschiedener Fachgesellschaften.

\section{Literatur}

[1] De Souza M], Williams NI, Nattiv A et al. Misunderstanding the female athlete triad: Refuting the IOC consensus statement on Relative Energy Deficiency in Sport (RED-S). Br J Sports Med 2014; 48(20): 1461-5

[2] Heikura IA, Uusitalo ALT, Stellingwerff T et al. Low energy availability is difficult to assess but outcomes have large impact on bone injury rates in elite distance athletes. Int Sport Nutr Exerc Metab 2018; 28(4): 403-411

[3] Kim JH, Malhotra R, Chiampas G et al. Race Associated Cardiac Arrest Event Registry (RACER) Study Group. Cardiac arrest during long-distance running races. NEJM 2012; 366(2): $130-40$

[4] Mountjoy M, Sundgot-Borgen JK, Burke LM et al. IOC consensus statement on Relative Energy Deficiency in Sport (RED-S): 2018 update. Br J Sports Med 2018; 52(11): 687-697

[5] Otis CL, Drinkwater B, Johnson M et al. American College of Sports Medicine position stand. The Female Athlete Triad. Med Sci Sports Exerc 1997; 29(5): i-ix

Bibliografie

DOI https://doi.org/10.1055/a-1130-9574

Sportphysio 2020; 8: 78-81

(c) Georg Thieme Verlag KG Stuttgart · New York ISSN 2196-5951

\section{Korrespondenzadresse}

\section{Dr. med. Christine Kopp}

Universität Tübingen

Hoppe-Seyler-Straße 6

72076 Tübingen

Christine.kopp@med.uni-tuebingen.de 